\title{
ACERCA DE LA TOPICALIDAD DE OBJETO EN ALGUNOS DIALECTOS DEL ESPAÑOL
}

\author{
Amparo Morales \\ Universidad de Puerto Rico
}

Hoy, con mayor seguridad que nunca, con el fructífero resurgimiento de la gramática funcional, que hace ya algunos años había propuesto la lingüistica praguense y que en nuestros dias ha remozado con éxito la pragmática y la gramática del discurso ${ }^{1}$, se corrobora que nuestras expresiones están organizadas de acuerdo con patrones que, más allá de los modelos formales - de unidades y combinaciones - reflejan explícitamente los significados y las intenciones comunicativas de los participantes de la conversación. Se ha comprobado que, en ocasiones, una misma función comunicativa puede llevarse a cabo por diferentes estructuras sintácticas, y al revés. Analizar cómo la estructura y uso del lenguaje coexisten y se desarrollan, medir hasta qué punto el significado referencial y comunicativo del mensaje que se emite se ve afectado por cambios en la estructura formal, es, tal vez, el interés primordial de la gramática actual.

Las fuerzas comunicativas que subyacen al manejo y ordenamiento de las estructuras sintácticas se reflejan en los hechos recurrentes que presen-

1 Obras ya clásicas en esa dirección son las de Wallace Chafe, «Givenness, contras. tiveness, definiteness, subjects, topics and point of view», en Subject and topic, editada por Charles Li, New York, Academic Press, 1976, págs. 25-36; «Cognitive constraint on information flow», en Coberence and grounding in discourse, editada por Russell Tomlin, Amsterdam \& Philadelphia, John Benjamins, 1987, págs. 21-51 y The pear stories: Cognitive, cultural, and Linguistic aspects of narrative production, Norwood, NJ, Ablex, 1980, de la que Chafe es editor. En general todos los artículos de Subject and topic. Además Michael A. K. Halliday, «Notes on transitivity and theme in English», Parte 1, 2 y 3, Journal of Linguistics, 3.1, 3.2 y 4.2, 1967/68, págs. 37-81, 199-244 у 179-215; у An introduction to functional grammar, London, Edward Arnold. Importantes son las publicaciones colectivas de Talmy Givón, Syntax and Semantics, vol. 12, New York, Academic Press, 1979, y John Haiman, Iconicity in Sintax, Amsterdam \& Philadelphia, John Benjamins, 1985. 
tan las lenguas - patrones de orden de las unidades en la cadena hablada, asignación de funciones a clases, posibilidad de elisión o pronominalización de algunas de ellas, etc.-, hechos recurrentes que pertenecen a la norma de realización del sistema y que dependen de las funciones comunicativas de sus unidades. Algunos hablantes, y con ello algunos dialectos del sistema general, pueden tener preferencias formales a la hora de expresar un significado común. Preferencias que, en raras ocasiones, desgraciadamente para los estudios de variación sintáctica, se ven desprovistos de repercusiones significativas ${ }^{2}$, especialmente si consideramos que la competencia comunicativa incluye un abanico muy amplio de significados posibles: intenciones comunicativas, modalidades de estilo, niveles de información compartida, etc. Con ello el dialectólogo inclinado hacia análisis sintácticos se encuentra, en nuestros días, ante un gran dilema, por un lado observa diferencias sintácticas dialectales que le gustaría constatar y, por otro, se le hace difícil, en términos metodológicos, acercarse a ellas. Nosotros consideramos, y en esto creemos hacernos eco de otros estudiosos ${ }^{3}$, que todo depende de la interpretación y alcance que los hechos analizados reciban.

Ofrecemos aquí la descripción de algunos de estos patrones sintácticopragmáticos del español, apoyándonos en los datos obtenidos en tres modalidades dialectales: el español de San Juan de Puerto Rico, el español porteño y el madrileño. Por encima de la interpretación que demos a los fenómenos analizados, la gran verdad es que sólo un acercamiento flexible a los hechos permite describir los rasgos particulares que configuran los patrones recurrentes de que hablábamos.

1. Hace apenas unos años ${ }^{4}$ llamamos la atención sobre determinados datos de variación sintáctica que parecían indicar tendencias diferentes en los patrones de transitividad de estos dialectos. Se trataba de estructuras sintácticas diferentes que formalizaban la misma función comunicativa y que mostraban diferencias entre los dialectos considerados. Nos referimos a la

2 Ralph Fasold ha sido, tal vez, el más categorico ante estos hechos, negando la posibilidad de que la variación sintáctica pueda darse, en The Sociolinguistics of Society, Oxford, Blackwell, 1984, con reimpresión en 1987. Una visión opuesta, o matizada, se encuentra en David Sankoff, «Sociolinguistics and syntactic variation», en Linguistics: The Cambridge Survey IV. Language: The socio-cultural Context, editado por Frederick J. Newmeyer, Cambridge, Cambridge Univ. Press, págs. 140-161. Se ofrece interpretación objetiva de todos estos planteamientos en Humberto López Morales, Sociolingǘfstica, Madrid, Gredos, 1989.

3 Nos referimos a la posición de Beatriz Lavandera en $*$ Where does the sociolinguistic variable stop?», Language in Society, 7, 1978, págs. 171-183.

- Amparo Morales, «Topicalidad y transitividad en algunos dialectos del español». Ponencia plenaria presentada al IX Congreso Internacional de la Asociación de Lingülstica y Filología de la América Latina, Campinas, Brasil, 1990. 
construcción de oración activa con objeto antepuesto y de la intransitiva pasiva refleja con la $\mathrm{FN}$ en primera posición. En ambas el paciente está antepuesto y parece desempeñar una función comunicativa similar: conector del discurso precedente. En ambas, el paciente, que ofrece información ya compartida o fácilmente accesible al oyente, sirve de marco de referencia para la nueva información que se ofrece. Los ejemplos que presentamos a continuación son casos claros de estas estructuras:

A. «los papeles los reciclan (desde hace unos años)».

B. «los papeles se reciclan (desde hace unos años)».

El análisis que ofrecemos aquí se basa en una muestra ampliada y depurada. En este caso se han tenido en cuenta todas las entrevistas de "diálogo dirigido" de la Norma Culta de Madrid y Buenos Aires. De las de Puerto Rico se eliminaron las entrevistas número IV, VIII, X y XII, por considerar que presentaban menos texto dialogado que el resto y ello podría implicar que se trataba de otro tipo de discurso. Estas entrevistas aparecen ya publicadas en sus volúmenes correspondientes y presentan un discurso dialogado entre entrevistados e informante similar en los tres textos ${ }^{5}$.

2. Tradicionalmente la pasiva con se se ha considerado una variante de la forma activa ${ }^{6}$. Variante que utiliza el hablante cuando no "interesa el agente" ?. Alcina y Blecua señalan el carácter inanimado del sujeto de estas estructuras y de la tendencia que manifiestan a colocar la frase nominal -paciente de la oración- pospuesta al verbo, "a diferencia de las de reflexivo medial que lo sitúan delante" 8 .

Siguiendo los planteamientos de Alcina y Blecua, respecto a que la pasiva refleja se utiliza cuando no hay interés en el agente y por lo tanto queda palidecida su referencia, los casos en que las transitivas activas de objeto

- Los textos son: M. Esgueva y M. Cantarero (eds.), El habla de la ciudad de Madrid. Materiales para su estudio, Madrid, C.S.I.C., 1981; Instituto de Filologia y Literaturas Hispánicas, El babla culta de la ciudad de Buenos Aires. Materiales para su estudio. Tomo I. Buenos Aires, Univ. Nacional de Buenos Aires, 1987; y Amparo Morales y Marfa Vaquero, El habla culta de San Juan. Materiales para su estudio, Río Piedras, Editorial de la UPR, 1990.

- En realidad, cuando se tiene en cuenta el discurso de los hablantes se observa que la función comunicativa que desempeñan es muy diferente. La pasiva sólo puede aparecer en los contextos en que el agente es el «foco* de la información que se emite.

7 Juan Alcina Franch y José Manuel Blecua nos dicen: «Cuando al hablante le interesa eliminar de la expresión al agente de la acción del verbo o pretende generalizar dicha acción sin especificar el agente, el castellano acude a la conversión de la estructura personal básica primaria en una especial estructura básica secundaria en tercera persona con 'se'» en Gramática española, Barcelona, Ariel, 1982 (1975, 1980), pág. 918.

- Alcina y Blecua, op. cit., 1982, pág. 919. 
antepuesto tengan un sujeto con referencia arbitraria presentarán una situación similar, en términos de las relaciones de agencia, a las primeras, ya que en ambas el agente queda opacado o disminuido. Esa característica es precisamente la que permite la aparición de la pasiva refleja sin agente expreso, en unas, y de una marca verbal de impersonalidad, como es la terminación de tercera persona plural $(-n)$, en otras.

Respecto a la función comunicativa, la pasiva con se no ha recibido mucha atención de los estudiosos, pero si partimos de la base de que la posición pospuesta de la FN es la neutral, como señalaban Alcina y Blecua, tendremos que postular que la anteposición es el orden marcado y, en ese caso, se utilizará por el hablante con determinado propósito comunicativo. Como la anteposición es, por excelencia, la posición de la topicalidad ${ }^{9}$, la función comunicativa del sujeto paciente de la oración intransitiva pasiva será, por lo menos en teoría, muy similar a la del objeto antepuesto de la transitiva. Esta interpretación es totalmente plausible y lógica, puesto que nos apoyamos en el hecho de que la pasiva con ser ofrece el sujeto paciente antepuesto porque no es él el foco de la información emitida, sino que lo es el agente que aparece pospuesto. A la FN de la pasiva con $s e$, por no tener ablativo agente, le corresponde la carga de la información y, de ahí, su posposición. Los casos de anteposición, como son los nuestros, responden a una situación comunicativa diferente.

3. La semejanza de las estructuras A y B parece reflejarse también en los ejemplos encontrados en nuestros textos; en ellos los hablantes en la misma situación comunicativa y ante un contexto de paciente topicalizado y agente no relevante, oscilan entre la expresión activa de objeto tópico y la pasiva con se de sujeto antepuesto. Así se ve en los siguientes casos que son párrafos continuos extraidos de nuestros materiales (los puntos suspensivos son pausas breves de los hablantes):

(1) «Las publicaciones... todo lo que se hacia en materia de geografia se preparaba y eso lo controlábamos aquí a través de la oficina. Todo eso lo vemos... eso se ficha y se guardaw (BA, XII).

(2) «En la Castellana habfa sillas para sentarse y esas se pagaban... Si las vendian en la... se vendian en la Puerta del Sol, muchas baratijas se vendian asis (M, XV).

(3) «Entonces hablamos un... grupo de profesores que decíamos esto hay que pararlo, pero no sablamos cómo, porque no se podia... porque no... no habla un lideratow (SJ, I).

- Véase Chafe, op. cit., 1976; Halliday, op. cit., 1985, y Talmy Givón, On Understanding Grammar, New York, Academic Press, 1979; por citar algunas de las obras más representativas que han trabajado con el concepto. 
Como se demuestra en estos ejemplos contrastivos, la posibilidad de cambio de una y otra estructura obedece a que el contexto tiene suficientes "agarres comunicativos" para mantener el significado por encima de estas ligeras variantes formales. Son "contextos restringidos" que permiten continuar con el mismo significado referencial de la agencia, el mayor palidecimiento agencial que presenta la estructura con se se neutraliza en el contexto.

Pronto nos dimos cuenta que en las oraciones activas transitivas tenian que incluirse no sólo las que aparecian con la marca de indeterminación de tercera personal plural $(-n)$, sino impersonales sin marca de persona como hay que $\mathrm{e}$, incluso, algunas de las que tenían como marca de indeterminación la flexión verbal de primera persona plural (-mos). En muchas ocasiones el hablante pertenece al grupo agencial indeterminado y este puede adquirir matices más o menos ambiguos de arbitrariedad ${ }^{10}$. Este hecho pudimos comprobarlo en un trabajo sobre el se impersonal en el cual el hablante parecía incluirse o excluirse, según contextos, de la referencia arbitraria ${ }^{11}$. El uso mayoritario, en el caso presente, fue la exclusión con el verbo en tercera persona plural. Los ejemplos encontrados de -mos se mueven desde un "nosotros" igual a "la humanidad", hasta uno con el significado del "grupo en el que me sitúo". En todos estos casos se mantiene el mismo significado referencial de la agencia cuando la oración se convierte en pasiva refleja. Igualmente algunas expresiones de pasiva con se favorecian el paso a -mos más que a $-n$. La oración siguiente es buen ejemplo de ello:

(4) «Entonces habíamos un grupo de profesores que... que... decíamos esto hay que pararlo, pero no sabíamos cómo porque no se podía... porque no habla un liderato. $\mathrm{O}$ sea, eso... esto no respondía a nada, era una cosa individual, de que a mí me tiraron una piedra yo voy a tirar una y... en eso hemos progresado porque ya en los últimos meses ya hemos visto que se pueden controlar algunas cosas, pero en aquella época aquello no se podia controlar.... (SJ, I).

Sin lugar a dudas, aquello no lo podíamos controlar hubiera sido mejor alternativa que aquello no lo podían controlar para aquello no se podía controlar. El hablante en este ejemplo usa las formas verbales de tercera per-

10 Lo cual quiere decir que este valor puede variar desde un nosotros que identifica un grupo nombrado con anterioridad, pero indeterminado en su composición, o, incluso, de aparición esporádica con el significado de «los de este país» o «los que nos encontrábamos ahl»; hasta un nosotros que señala a unas personas especificas. Lo importante para nuestro análisis es que en el primer caso permite igualmente la forma con se y el significado no se altera.

${ }_{11} \mathrm{En} * \mathrm{El}$ pronombre indefinido uno en sus valores impersonales. Nuevos acercamientos», que aparecerá en Voz y Letra III-1, 1992, ofreciamos los datos respecto al se impersonal. Ellos demostraban que en muchas ocasiones el hablante se incluía en el grupo referencial indeterminado del se e, incluso, podfa alternar con uno. 
sona (se pueden controlar algunas cosas), primera plural (deciamos, sabiamos, hemos visto), se (no se podia, aquello no se podía controlar) e impersonal verbal (hay que pararlo) con referentes muy similares, especialmente las tres últimas (primera persona plural, se e impersonal verbal) que se relacionan con "aquella época" y la posición del profesorado entonces. Las diferencias entre las formas personales y las de se estriban en lo que hemos llamado un mayor "palidecimiento" de la agencia y eso es justo lo que se propone medir este trabajo, las preferencias por formas más o menos opacas en cuanto al agente en contextos "restringidos" 12.

Los cambios de forma verbal en la oración (3), que se repiten en (4), son muy ilustrativos. Tanto esto hay que pararlo como (esto) no se podia (parar) tienen como agente de la acción al grupo indeterminado de profesores observadores de los hechos, la única diferencia es la que aportan las formas mismas en términos de la obligatoriedad de que la acción se lleve a cabo, pero también aqui el hablante podia haber recurrido a una forma sustituta de obligación, esto se tiene que parar como alternativa de esto hay que pararlo, $\mathrm{y}$, claro, también esto tenemos que pararlo.

3.1. El análisis de topicalidad, según la escala de Prince o variantes ${ }^{13}$, nos permitió establecer cuatro categorías en la FN antepuesta. Estas categorías, que se graduaron de acuerdo con el tipo de información que aportaban, quedaron representadas, en nuestro caso, en cuatro niveles de "información compartida" vs. "novedad".

La categoría (a) incluía los "conocidos", representados por las frases nominales que se hubieran podido eliminar sin perder información, como el ejemplo (5):

(5) «Por ejemplo, tenemos una propuesta de crear un departamento de an-

12 Una definición provisional de *contexto restringidow para estas formas serfa *aquellos en los cuales el cambio de forma no conllevs cambio de referentew.

1s La primera interpretación de valores graduados y formalizados en categorfas aparece en Ellen Prince, *Toward a taxonomy of given-new information», en Radical pragmatics, editado por Peter Cole, New York, Academic Press, 1981, págs. 223-255. En esta «escala de familiaridad los valores van desde los más conocidos (evoked, unused, inferable) $\mathrm{y}$, con ello, con más posibilidades de ser tópico, a los más nuevos (brand-new anchored y brand-new). Posteriormente se han propuesto otras categorfas que tienen que ver con la mayor o menor posibilidad de *identificación» y la «actividad» que haya mostrado la entidad en el texto precedente (Knud Lambrecht, \&Presentational cleft constructions in spoken French, en Clause combining in grammar and discourse, editado por John Haiman y Sandra Thompson, Amsterdam \& Philadelphia, John Benjamins, 1988, págs. 135-179; y Paola Bentivoglio, «Full NPs in spoken Spanish: A discourse profile», aparecerá en Papers from the XXI Linguistic Symposium on Romance Languages, Santa Bárbara, 1991). Estos articulos no agotan el tema, desde luego; interpretaciones relacionedas aparecen en Chafe, 1987, op. cit., y John Du Bois W., *Beyond definiteness: The trace of identity in discourse», en Chafe, 1980, op. cit., págs. 203-274. 
tropología en la cual no habrá prerrequisito, ni cursos introductorios, y abierto a cualquier estudiante de la Universidad de Puerto Rico porque creemos que eso se puede hacer...» (SJ, I).

(6) «entonces ese archivo mismo se vuelve a grabar en otra cinta» (BA, XII).

(7) «porque México aqui lo vemos como el bastión de la libertad» (SJ, ?).

La categoría (b) eran evocados o nombrados previamente, pero necesitaban volver a nombrarse para entender el mensaje o porque la situación comunicativa requería la repetición ${ }^{14}$. La mayoría de los casos en que la FN era un demostrativo neutro cayó en esta categoría:

(8) «Sí un centro ceremonial que utilizaban los indios para... (- ¿y que se descubrio hace poco?-). No, no, esto se descubri6, mire, cuando llegaron los americanos»

(SJ, VI).

(9) «que yo creo que ambos cursos se deberian unir en uno» (SJ, II).

(10) «(-cY el $Y a ?-)$.

El Ya lo leíamos en casa los domingos» (M, IV).

La categoría (c) incluía las frases nominales que estaban implicadas, pero no nombradas en la categoría anterior, eran elementos que pertenecían a un conjunto anteriormente nombrado y que se añadian al mismo, o completaban una apreciación más abstracta :

(11) «hasta el punto que una cuestión de reformar un curso, o un departamento, se entiende en términos de quiénes están propulsando eso» (SJ, I).

(12) «lo que está ocurriendo en el mundo bay que recibirlo» (BA, IX).

(13) «los reproductores los compramos» (BA, XVI).

A la categoría (d) pertenecian las más nuevas o contrastivas, especialmente se incluyeron aquí los casos de "foco de contraste" y "contrario a lo esperado" que nombra la bibliografía tradicionalmente ${ }^{15}$ :

(14) «Ya en los últimos meses ya hemos visto que se pueden controlar algunas cosas, pero en aquella época, aquello no se podía controlar» (SJ,

\footnotetext{
14 En la gradación de unidades por nivel de «familiaridad» parecen intervenir dos factores diferentes, por un lado el grado de novedad que el elemento aporte y, por otro, las funciones pragmáticas asignadas (Francisco Ocampo, «The pragmatics of word order», Hispanic Linguistics, 4:1, 1990, págs. 87-128).

15 Estas hacen referencia directamente a funciones pragmáticas (Ocampo, op. cit., 1990). Para una descripción de ellas y los aspectos relacionados con la topicalidad, véase Carmen Silva-Corvalán, Sociolingüistica. Teoria y práctica, Madrid, Alhambra, 1989.
} 
(15) wlas bembras se reservan para ordenarles y los machos se castran para engordarlos* (BA, VIII).

Los resultados aparecen en el cuadro 1. Tanto en A, como en B, la frase nominal antepuesta ofreció distribución muy similar en las categorías establecidas. El peso recae en la categoria (b), que obtuvo un $57.14 \%$ en A (construcción activa con objeto antepuesto) y un $55.72 \%$ en $\mathrm{B}$ (construcción pasiva refleja). Como se esperaba, por ser contextos de topicalidad, las frases nominales antepuestas no mostraron valores elevados de "novedad", se mantuvieron mayormente en la categoría de "conocido" o "evocado" que equivalen a las (a), (b) y (c) de nuestro análisis.

Cundro 1

FNs antepuestas según escala de topicalidad

\begin{tabular}{|c|c|c|c|c|c|c|c|c|}
\hline & \multicolumn{4}{|c|}{ A } & \multicolumn{4}{|c|}{ B } \\
\hline & (a) & (b) & (c) & (d) & (a) & (b) & (c) & (d) \\
\hline Madrid & 2 & 14 & 4 & - & 8 & 21 & 8 & 1 \\
\hline B. Aires & 5 & 12 & 8 & 4 & 4 & 12 & 4 & 3 \\
\hline San Juan & 7 & 22 & 6 & - & 16 & 40 & 14 & - \\
\hline Totales: & 14 & 48 & 18 & 4 & 28 & 73 & 26 & 4 \\
\hline
\end{tabular}

3.2. Por otro lado, se calculó la representatividad de las frases nominales antepuestas según sus rasgos formales: Pronombres, Demostrativos neutros (PronN en el cuadro), FNs definidas, FNs indefinidas y Otros. Los resultados aparecen en el cuadro 2 :

Cuadro 2

FNs antepuestas según clases formales

\begin{tabular}{|c|c|c|c|c|c|c|c|c|c|c|}
\hline & \multicolumn{5}{|c|}{ A } & \multicolumn{5}{|c|}{ B } \\
\hline & Pron & PronN & FNDef & FNInd & Otro & Pron & PronN & FNDef & FNInd & Otro \\
\hline Madrid & - & 3 & 15 & 1 & 1 & 3 & 6 & 26 & 3 & - \\
\hline B. Aires & 1 & 4 & 18 & 4 & 2 & 1 & 7 & 13 & 1 & 1 \\
\hline San Juan & 1 & 14 & 20 & - & - & - & 28 & 38 & 3 & 1 \\
\hline Totales: & 2 & 21 & 53 & 5 & 3 & 4 & 41 & 77 & 7 & 2 \\
\hline
\end{tabular}


Como en el caso anterior, la distribución por categorías fue muy similar, los demostrativos neutros y las frases nominales definidas fueron las más representadas tanto en $\mathrm{A}$, como en $\mathrm{B}$. Un $25 \%$ de pronombres neutros frente a $63 \%$ de frases nominales definidas en $\mathrm{A}$ y un $31 \%$ frente a $59 \%$ en $\mathrm{B}$.

La mayoria de frases nominales antepuestas fueron no humanas, con ligera preferencia por ellas en las construcciones transitivas. De los 84 objetos antepuestos $14(9,52 \%)$ tenían el rasgo + humano y $8(6.10 \%)$, de los 131 sujetos de las construcciones con $s e$.

Esta semejanza en los datos cuantitativos no hace sino confirmar los hechos observados en las entrevistas, las situaciones comunicativas son muy similares en todos los casos, responden al mismo patrón: selección de un elemento del contexto anterior, o relacionado con el contexto anterior, para respecto a él añadir nueva información, en una situación en la cual, aunque haya agencia externa implícita, esta no se conoce o no es relevante. En todas ellas el contexto fue suficientemente explícito para mantener la misma interpretación, aunque se efectuara un cambio de estructura (A por B, o viceversa).

Por otro lado, debido a que sólo se consideraron las estructuras que permitían el cambio de una construcción por otra, las seleccionadas tenían rasgos semánticos similares — que eran los que permitían el cambio- y que venían dados por los rasgos del mismo verbo. Efectivamente, los verbos eran de los llamados resultativos ${ }^{16}$, que tenían implícito un agente externo indeterminado con acción que permitía un fin. No se habian incluido, por ello, los casos en que la forma pasiva producía una interpretación seudorreflexiva o media, como sucede en los ejemplos siguientes:

(16) «los problemas en el hospital militar se resolvian por sí solos» (SJ, XXII).

(17) «no hay una estructura que permita que esto se siga dando en gran es. cala» (SJ, VI).

3.3. La comparación dialectal de estas estructuras se efectuó en tres contextos diferentes: las construcciones A y B, ya analizadas y dos contextos relacionados, los de objeto representado por un clítico y el de oración relativa de objeto representado por un clítico y el de oración relativa de objeto. En ambos se repite la misma posibilidad de alternancia que observamos en las de objeto antepuesto.

16 Véase Halliday, op. cit., 1985, pág. 149. Cuando se trata de lenguas que marcan casos estos diferentes patrones sintácticos se ofrecen como dos estructuras alternas que presentan las lenguas que pertenecen a distinta clasificación tipológica: ergativas-absolutivas y nominativas-acusativas (Bernard Comrie, Language Universals and Linguistic Ty. pology, Chicago, Univ. of Chicago Press, 1981). El español es una lengua nominativoacusativa, aquil se trata de patrones de realización en determinados contextos. 
Los datos del primer contexto aparecen en el cuadro 3. Las diferencias en términos porcentuales muestran preferencia por parte del dialecto porteño a la construcción activa.

Cundro 3

Construcciones de objeto antepuesto

\begin{tabular}{llll}
\hline & \multicolumn{1}{c}{ FN + cli. } & & se \\
\cline { 2 - 2 } Madrid & $20(34.5 \%)$ & & 38 \\
B. Aires & $29(56 \%)$ & & 23 \\
San Juan & $35(33.3 \%)$ & & 70 \\
\hline
\end{tabular}

p. $<0.10$

El otro contexto medido fue el de las oraciones de relativo objeto en las cuales al hablante se le presenta la misma posibilidad de hacer del relativo el objeto o el sujeto de la expresión siguiente:

(18) wera uno de esos coches que se..., que los contratan porque en el verano hay escasez* (M, XVI).

de hecho, como demuestra la oración (18), el hablante tiene otra nueva opción, la de duplicar en la construcción activa el relativo con un clítico. Los casos encontrados responden a los tres contextos siguientes:

(19) «quieren que el RTC sea voluntario, que lo logran» (SJ, I).

(20) «ese es un salón que se diseñó como salón de clases» (SJ, II).

(21) «las cualificaciones que pedian eran esas» (SJ, IX).

Las diferencias dialectales en estos contextos no fueron relevantes, como se puede apreciar en el cuadro 4 :

\section{Cundro 4}

Construcciones de relativo objeto

\begin{tabular}{lccccc}
\hline & rel. + cli. & & rel. & & \multicolumn{1}{c}{ rel. + se } \\
\cline { 2 - 2 } \cline { 5 - 5 } \cline { 5 - 6 } Madrid & 8 & & 20 & & 34 \\
B. Aires & 6 & & 11 & & 23 \\
San Juan & 9 & & 39 & & 66 \\
\hline
\end{tabular}

La diferencia más importante, teniendo en cuenta la escasa representación numérica, fue la realización de relativo objeto más clítico que en el 
RFE, LXXII, 1992 TOPICALIDAd DE OBJETo EN ALGUNOS DIALECTOS DEL ESPAÑoL 681

dialecto porteño alcanzó la manifestación más alta: $35.29 \%$ en BA, $28.57 \%$ en M y $18.75 \%$ en SJ. Volveremos a esto más adelante.

El tercer grupo medía las diferencias de transitividad en contextos más neutrales en términos comunicativos, son los que presentan un objeto ya nombrado que aparece pronominalizado. También en él se dan las mismas circunstancias de alternancia.

Los casos analizados responden a los ejemplos que aparecen a continuación :

(22) «cualquier incidente hace que esto explote, entonces no tiene control, no, no se puede controlar» (SJ, I).

(23) «Me llevaba en el treinta y uno; lo tomábamos en la esquina de Pueyrredón y Santa Fe» (BA, III).

Los resultados se encuentran en el cuadro 5 que, aunque muestran ciertas diferencias, preferencia por la estructura activa por parte de la modalidad bonaerense, estas no fueron significativas.

Cundro 5

Pronominalización de objeto

\begin{tabular}{lrrr}
\hline & \multicolumn{1}{c}{ cli. } & & se \\
\cline { 2 - 2 } Madrid & $57(68 \%)$ & & 27 \\
B. Aires & $87(77 \%)$ & & 26 \\
S. Juan & $100(68 \%)$ & & 47 \\
\hline
\end{tabular}

3.4. En todas estas estructuras A y B se encuentran también casos en que el hablante utiliza el se y un clítico correferente con el objeto directo o indirecto. En estas situaciones la interpretación del se es obligatoriamente de se impersonal y la construcción pasa a ser activa. Nos referimos a casos como los siguientes:

(24) «la sociedad, si hay que cambiarla, se la puede cambiar» (M, I).

(25) «después, otro niño alemán, que tampoco se le ba encontrado» (M, III).

(26) «primero se lo lleva en camiones hasta Mendoza» (BA, VIII).

En los tres contextos analizados aparece este tipo de casos, algunos de ellos ya agramaticales como es la oración (26) que no cumple la restricción del español que impide la aparición de un clítico masculino unido al se im- 
personal ${ }^{17}$. En todos los casos es posible el cambio a pasiva refleja -eliminando el clítico- y a la construcción activa (eliminando el se y poniendo el verbo en tercera persona o primera plural).

Vuelve a ser aquí la variedad bonaerense la que más casos presenta de este tipo, incluso con construcciones agramaticales para otros dialectos, como el ejemplo (26) que tiene el clítico en acusativo masculino ${ }^{18}$. Los datos son los siguientes: 12 de los 204 casos totales manejados en la modalidad madrileña, 16 de 205 en la porteña y 13 de 366 en la puertorriqueña. Los dialectos puertorriqueño y madrileño no presentaron ningún caso de se impersonal con clítico masculino, los de San Juan todos eran le o les:

(27) *cuando se les ocupa» (SJ, XI).

(28) «también se les premia con dinero" (SJ, XIV).

(29) *eso se le decía delito menos grave» (SJ, VII).

en Madrid se encontraron casos de clítico femenino.

4. El análisis quedaría incompleto si no añadiéramos los datos generales obtenidos en investigaciones anteriores nuestras sobre la duplicación con clítico del objeto pospuesto y del relativo objeto.

Ya Jaggli, en su trabajo sobre las lenguas romances ${ }^{19}$, habia señalado ciertas particularidades del español porteño. Este difería del modelo general del uso de los clíticos por su tendencia a duplicar el objeto. Se refería a la llamada conjugación objetiva, comentada ya por varios estudiosos ${ }^{20}$.

El mayor problema cuando se quieren probar este tipo de hechos es que la representatividad numérica es mínima, sin lugar a dudas la variación dialectal de esta clase de rasgos es limitada. Pero nuestros datos numéricos,

\footnotetext{
17 Hacen alusión a esa restricción del español: Franco D'Introno en «Alternancia lo/le en el español de Caracas», en Corrientes actuales de la dialectologia del Caribe bispánico, editado por Humberto López Morales, San Juan, Editorial Universitaria, 1978, págs. 51-76, y Marina Fernández Lagunilla en *Acerca de la secuencia 'se impersonal + enclítico de tercera persona' ¿una restricción superficial?», Revista Española de Lingüística, 5:1, 1975, págs. 177-195.

18 En conversación personal con Beatriz Fontanella en Campinas, nos informó que efectivamente a ella le parecían expresiones corrientes que estaba acostumbrada a ofr en su pals, Argentina.

10 El autor presenta un modelo teórico para la interpretación de los hechos en Osvaldo Jaeggli, Topics in Romance Syntax, Dordrecht, Foris Pub., 1982.

${ }^{20}$ Para una visión global y muy documentada del fenómeno: Antonio Llorente y J. Mondéjar, «La conjugación objetiva en español», Revista Española de Lingüfstica, 4, 1974, págs. 1-60.
} 
dentro de sus limitaciones, mostraban que había, efectivamente, unas tendencias que podían tenerse en cuenta si formaban parte de otro conjunto de datos configurativos. Los ejemplos son los siguientes:

(30) «no lo he llegado a tener nunca un diálogo inteligente» (BA, VII).

(31) «yo de marino no lo he conocido nunca a mi padre (M, XV).

(32) «y las veía las obras montadas» (SJ, VI).

Repetimos aquí los números, que son constancia de la poca representatividad que tiene aún el fenómeno en el español.

\section{CUADRo 6}

Realización de clf. con OD pospuesto

\begin{tabular}{lll}
\hline Madrid & $19 / 1030$ & $1.81 \%$ \\
B. Aires & $33 / 1464$ & $2.20 \%$ \\
San Juan & $11 / 1666$ & $0.65 \%$ \\
\hline $\mathrm{p}<0.001$ & &
\end{tabular}

Por otro lado, la modalidad porteña ofrece más casos de oraciones de relativo objeto, en general -no sólo las consideradas en estructuras A y B- en las que el relativo aparece duplicado con una forma pronominal, la tendencia a la duplicación con clítico, que vimos en los casos de relativas A, se mantiene en el conteo general. Se trata de casos como los siguientes:

(33) «a Pérez de Ayala que lo vefa todas las semanas» (M, XVI).

(34) «fue una película que la tomó en sus manos Omar Shariff» (SJ, VI).

(35) «tienen una alta calidad que la han logrado» (BA, VIII).

cuyos resultados aparecen en el cuadro 7 .

\section{Cundro 7}

Oraciones de relativo objeto

\begin{tabular}{lrrrr}
\hline & rel. + cli. & & \multicolumn{1}{c}{$\%$} \\
\cline { 2 - 2 } Madrid & $8 / 74$ & & $6.50 \%$ \\
B. Aires & $45 / 104$ & & $24.45 \%$ \\
S. Juan & $28 / 122$ & & $14 \%$ \\
\hline p $<0.001$ & & &
\end{tabular}


5. De las comparaciones presentadas en este trabajo han resultado significativas las que medían los contextos de objeto antepuesto (A vs. B), y las generales de duplicación con clítico del relativo objeto $\mathrm{y}$ duplicación de objeto pospuesto. No lo fueron la comparación de las estructuras A y B en oraciones de relativo objeto y de objeto pronominalizado.

Estos resultados, por el momento, indican que las diferencias se apoyan más en los rasgos relacionados con las marcas de concordancia de objeto que en los de transitividad propiamente. Así las diferencias entre A y B son relevantes únicamente cuando A tiene que ir acompanada de un clítico redundante (anteposición de objeto), y no, en los casos de simple oposición transitiva vs. intransitiva (objeto pronominalizado u oración de relativo).

En las oraciones de relativo, los hablantes de Buenos Aires no construyen más oraciones de relativo objeto transitivas (A vs. B) que los otros dialectos, pero sí presentan más veces clíticos que duplican al relativo objeto, igualmente lo hacen con el objeto en posición neutral. Esa tendencia a la concordancia de objeto es la que caracteriza a la modalidad porteña frente a la madrileña y sanjuanera.

Respecto a las estructuras analizadas A y B, se ha insistido en la mayor formalidad que comunica al mensaje la forma se y que, por ello, podría haber diferencias de estilo, mayor o menor formalidad, entre los tres corpora de análisis. Hay que tener en cuenta que estas características de formalidad del se responden más al orden neutral, en el que las FNs pospuestas aportan información nueva y son foco del mensaje, ello las hace configurar un discurso con mayor carga informativa y más adecuado para la exposición formal. La anteposición responde a una situación comunicativa diferente, de estilo más coloquial. Por otro lado, junto al mayor uso de construcciones activas de A, por parte de la modalidad bonaerense, se da también, en este dialecto, la mayor aparición de se impersomal con clítico acusativo masculino, preferencia que pudiera ir en contra de estas consideraciones sobre la formalidad.

Como decíamos anteriormente, las estructuras A y B presentan ciertas diferencias, diferencias relacionadas con la opacidad del agente, la B (estructura con se) parece alejarse un tanto más de este. La marca de persona en el verbo en la estructura A permite interpretar con un grado mayor de intensidad la participación de dos argumentos (sujeto y objeto), esto comunica a la construcción un grado mayor de transitividad ${ }^{21}$. Esas diferencias son las que ocasionan las distintas variedades dialectales, cierta particular modalidad del discurso, que viene dada por la preferencia de una u otra forma en los contextos restringidos.

n Véase Paul J. Hopper y Sandra A. Thompson, *Transitivity in grammar and discoursew, Language, 56:2, 1980, págs. 251-299. 
Si se hubiera comprobado que las diferencias entre A y B se mantenian en todos los contextos analizados, no sólo en los de objeto antepuesto, podríamos suponer que se trataba de variaciones más generales; con nuestros resultados sólo podemos decir que si pensamos que la conjugación objetiva puede tener manifestaciones de sólo tendencias, sin duda es el dialecto porteño el más afectado por esas tendencias en el sistema general del español. 